\title{
Cognitive Behavioral Training Reduces Socially Anxious Classroom Behavior in Primary School Students
}

\author{
Gino Casale ${ }^{1 *}$ and Daniel Haarhoff ${ }^{2}$ \\ ${ }^{1}$ Methods and Didactics in Special Education, Institute for Educational Research, School of Education, University of Wuppertal, \\ Wuppertal, Germany, ${ }^{2}$ Institute for Educational Sciences, University of Paderborn, Paderborn, Germany
}

Many students show symptoms of social anxiety. Cognitive behavioral training (CBT) has been shown to be effective in reducing symptom behavior in therapeutic and school contexts, but there are hardly any single-case studies that examine the individual effectiveness in school settings. Furthermore, the extent to which differential responsivity effects are exhibited by students has not been examined yet. This singlecase study with $A B$ design investigates the effects of a CBT on the socially anxious classroom behavior of students with severe symptoms of social anxiety. Two female and two male students (9-10 years old) of an inclusive primary school in Germany participated in the CBT over a period of twelve weeks. Socially anxious behaviors were measured daily

OPEN ACCESS

Edited by:

Joseph Mintz,

University College London,

United Kingdom

Reviewed by:

Lilly Augustine,

Jönköping University, Sweden

Ariel Mariah Lindorff,

University of Oxford, United Kingdom

*Correspondence:

Gino Casale

gcasale@uni-wuppertal.de

Specialty section:

This article was submitted to

Special Educational Needs,

a section of the journal

Frontiers in Education

Received: 23 July 2021 Accepted: 13 January 2022

Published: 08 February 2022

Citation:

Casale $G$ and Haarhoff D (2022) Cognitive Behavioral Training Reduces Socially Anxious Classroom Behavior

in Primary School Students.

Front. Educ. 7:746094.

doi: 10.3389/feduc.2022.746094 with Direct Behavior Rating-Multi Item Scales. Visual analyses, overlap indices, and regression models show a substantial behavioral improvement for all students during the intervention. However, there are differences between the students in terms of which specific behaviors were improved and whether the behavioral improvements took place immediately after the implementation of the intervention or continuously from measurement to measurement in the $\mathrm{B}$ phase. The results highlight the relevance of specific and individualized behavioral goals for CBT in school, although the effects should be replicated in experimental studies.

Keywords: cognitive behavioral training, social anxiety, classroom behavior, school-based intervention, single-case research

\section{INTRODUCTION}

Social anxiety is among the most prevalent emotional and behavioral disorders in school-aged children and youth with relatively early ages of onset and high rates of comorbidity with many others internalizing behavioral problems (Merikangas et al., 2010). Social anxiety is known to have a negative effect on the development of children and youth and is strongly associated with psychiatric disorders and substance abuse in adulthood, suicidal behavior, and educational underachievement (Woodard and Ferguson, 2001; Copeland et al., 2014). In school contexts, social anxiety interferes with several areas of functioning such as classroom participation, school achievement, or interaction with peers (see e.g., Cohen et al., 2019).

Cognitive behavioral training (CBT) "refers to a collection of therapeutic techniques and strategies that are used to alter behavior by teaching individuals to actively participate in understanding and modifying their own thoughts and behaviors" (Mayer et al., 2005, p. 197). This means that, in essence, CBT basically incorporates behavior modification strategies and 
cognitive modeling techniques. CBT for children and adolescents with social anxiety has been shown to effectively reduce symptoms of their disorder with moderate to large effects (Scaini et al., 2016). Schools offer a suitable setting for CBT for students with social anxiety, since the school context provides an excellent opportunity to generalize and practice the newlydeveloped skills in the children's everyday environment (see e.g., Domitrovich et al., 2010), and intervention effects have been found to be larger in school contexts (Scaini et al., 2016). Despite this potential of CBT, there is still a lack of controlled single-case studies that test patterns of individual students' responsiveness to CBT, especially with a focus on students who are not formally diagnosed with social anxiety disorders, but who exhibit severe clinical symptoms of social anxiety. Such studies are particularly important for working with students with emotional and behavioral disorders to expand the empirical evidence base on the effectiveness of CBT (see e.g., Gresham, 2005). Here, the use of controlled single-case research has at least four advantages: First, this methodological approach allows us to examine the response to an intervention of individual students or smaller groups of students with shared characteristics (Riley-Tilman et al., 2020). Second, single-case research allows capture of important idiosyncrasies of individual students that might explain intervention success (ibid.). Third, the repeated and close-meshed measurements in a baseline and intervention phase allow for a systematic comparison of developmental trajectories without and with intervention, as well as specific patterns of intervention effects, which in turn can be used to develop evidence-based support methods (Huitema and McKean, 2000). Fourth, the approach is highly feasible, especially for studies with small target populations (such as students with special education needs) (Maggin et al., 2018). In addition, data from multiple baseline designs should be analyzed inferentially to draw inferences regarding the individual patterns of students' responsiveness that might lead to important practical implications in intervention planning. In this regard, the single-case experimental research approach offers the opportunity to control for the manipulation of an independent variable and thus to examine the functional relationship between the independent and dependent variable more closely, as is uncommon in traditional case studies, for example.

To overcome this desideratum, we evaluated the individual response of four third-grade students with severe social anxiety symptoms to CBT with integrated social skills training using a multiple baseline single-case design. The training was implemented in an inclusive primary school in Germany, integrated into the students' daily school routine and conducted by an in-service special education teacher. Analysis of progress was done by calculating descriptive statistics, overlap effect sizes, and using piecewise-linear regression models for both all four and each individual student.

\subsection{Social Anxiety in School-Aged Children}

Social anxiety can be defined as a dimensional set of internalizing behavioral problems in social situations in which the individual is exposed to possible scrutiny by others (e.g., social interactions with peers, being observed while eating, or performing in front of peers; American Psychiatric Association APA, 2013; Rapee and Spence, 2004). These social situations provoke fear and anxiety in affected children and youth, which often results in an avoidance of these situations or in intense fear and anxiety in the situation (APA, 2013). Children and youth who exhibit social anxiety experience distress and impairment in several important areas of functioning, such as interactions with peers and school performance. Social anxiety often occurs comorbid with other anxiety disorders, depressive disorder, and substance abuse (Kessler et al., 2012).

Prevalence studies reveal rates of $5-7 \%$ of all school-aged children afflicted by social anxiety (see e.g., Kessler et al., 2012; Klasen et al., 2016). Most recently, the working group of the national BELLA cohort study in Germany published prevalence rate figures of $5-6 \%$ of all student whose parents reported symptoms of a social anxiety disorder (Klasen et al., 2016). Kessler et al. (2012) found 8.6\% (ages 13-17) of youth with social anxiety in a representative sample from the US National Comorbidity Survey with significantly higher rates in female participants. Furthermore, the persistence rates are high, and social anxiety often turns into a chronical disorder. Blanz et al. (2006) reported that approximately $45 \%$ of children with social anxiety continue to experience the symptoms throughout the course of their childhood until reaching adulthood.

Socially anxious behaviors have a negative effect on students' ability to function in school, these impairments may take the form of an inability to participate in class, poor achievements in school, or impaired peer interaction. For instance, students with social anxiety avoid talking during class discussions, do not participate in class or group activities, and are not able to process valuable information because they preoccupied with a fear of being judged by others (see e.g., Cohen et al., 2019). In addition, students with social anxiety often avoids peer interactions, and they are often socially excluded by their classmates (Krull et al., 2018), possibly leading to feelings of isolation and loneliness (Zhu et al., 2019).

A well-examined and highly stable predictor of social anxiety in children and adolescent is behavioral inhibition (BI), which is a temperament style occurring in infancy and early childhood (Clauss and Blackford, 2012; Luis-Joaquin et al., 2020; Sandstrom et al., 2020). BI is based on a cognitive-behavioral mechanism that manifests itself as an exaggerated cognitive sensitivity to novel auditive and visual stimuli, and an avoidance of unfamiliar situations and people (Clauss and Blackford, 2012; Fox et al., 2005; Kleberg et al., 2021). BI is closely linked to a dual model of information processing including automatic (e.g., novelty detection, attention biases to threat, and incentive processing) and controlled (e.g., attention shifting and inhibitory control) information processing (Henderson et al., 2015). Considering this predictor of social anxiety, treatment approaches for children and adolescents should focus on restructuring maladaptive cognitions in uncertain social situations and on rehearsing behavioral models that 
facilitate socially competent action. One treatment approach that incorporate these principles is cognitive behavioral training (CBT).

\subsection{Cognitive Behavioral Training for Students With Social Anxiety}

As already discussed above, Mayer et al. (2005) conceptualize CBT as an amalgamation of various therapeutic techniques and strategies for changing behavioral patterns. These techniques share the basic principle that maladaptive cognitions maintain behavioral disorders (Hofmann et al., 2012), and that "thoughts, emotions, and actions are inextricably linked and that changing one of these necessarily produces changes in the others" (Gresham, 2005, p. 213). Therefore, CBT targets the aforementioned cognitive-behavioral mechanisms of $\mathrm{BI}$ and dual information processing. It basically aims to change maladaptive cognitions and thereby also change problematic behaviors. CBT achieves this goal by combining cognitive modeling techniques, such as psychoeducation, cognitive restructuring, progressive muscle relaxation, and metacognitive strategy training, with behavior modification strategies, such as (self-)reinforcement, rewards, and feedback. In cases of social anxiety in particular, CBT frequently also makes use of social skills training to help children and adolescents interpret social situations appropriately and acquire skills for acting in a socially competent manner in these situations (Scaini et al., 2016).

The development and research of CBT for social anxiety in general and in particular for the school context is still quite young. This is mainly due to the fact that CBT originates from the field of clinical therapy and is more commonly used for the treatment of depressive disorders and generic anxiety disorders. However, CBT is also very well suited for use in the school context due to its basic conceptualization and the methods used, allowing students to experience in a natural school setting a variety of social situations in which socially competent action is required. The competencies acquired in CBT could thus be directly applied and generalized across contexts. In addition, students interact with numerous peers during the school day, so the group can be used as a resource for building up a robust repertoire of social skills. Finally, the material, and professional resources for effective mental health support are usually already present in school (see e.g., Domitrovich et al., 2010), since many schools offer the possibility to work with small groups in differentiation rooms, and several teachers often work in co-teaching in one class.

To consider CBT as an evidence-based intervention for students with social anxiety, it is important to examine the empirical support of its effectiveness in school contexts (see e.g., Gresham, 2005). In a meta-analysis of control group studies on the efficacy of CBT for social anxiety, Scaini et al. (2016) included a total of 13 studies that had a clear focus on CBT for children or adolescents (from 6 to 18 years of age) with social anxiety disorders. Regarding the school context, they found a strong effect of Hedge's $g=1.55$ on reducing symptoms of social anxiety disorder in pre-post studies. This effect was substantially higher than the overall effect in clinical contexts $(g=0.67$; Scaini et al., 2016). Similar effect sizes were found in a meta-analysis by Segool and Carlson (2008), which showed that treating school-aged children and adolescents with social anxiety with CBT resulted in strong reductions in its symptoms $(g=1.30)$, moderate reductions in symptoms of general anxiety $(g=0.75)$, and moderate improvements in social competencies. In a controlled single-subject study with multiple baselines across three six-grade students with emotional and behavioral disorders, Schoenfeld and Mathur (2009) implemented the FRIENDS curriculum, which is a CBT group intervention. Applying visual analysis and the Percentage of Nonoverlapping Data Points index (PND), they found a decrease in anxiety symptoms, and an improvement in academic engagement as well as in school-appropriate behavior (Schoenfeld and Mathur, 2009).

\section{DESIDERATA, RESEARCH QUESTIONS AND HYPOTHESES}

Considering the advantages and successes illustrated above, CBT appears as a potential approach for treating students with social anxiety in schools. Results from control-group and single-case studies provide empirical support for effective reduction of symptom behavior and improvement of social behaviors, though the amount of studies in school contexts is still sparse. We were only able to find one single case study examining the individual treatment responses of students with social anxiety in school contexts, and this study relies solely on visual and descriptive analyses. This is despite the high potential of single-case studies to bring added value, especially for schoolbased work with students with or at risk for behavioral disorders, as such studies usually allow us to infer the individual responsiveness to intervention and, therefore, to guide schoolbased interventions accordingly. In addition, experimental research has shown that visual analysis, especially for data with a baseline trend, lacks validity and is prone to a judgement bias in committing a Type 1 error (Wilbert et al., 2021). Researchers do, in fact, have an array of, advanced, regression-based approaches to the analysis of single-case data at their disposal that allow for a differentiated analysis of effect patterns in addition to an analysis of an overall intervention effect (Manolov and Moeyaert, 2017). This emphasized the necessity of conducting further controlled single-case studies to examine the individual responses of individual students with social anxiety to CBT in a differentiated manner. In addition to visual and descriptive analysis methods, these studies should also examine inferential statistics and control for potential baseline trends to determine whether or not there is an intervention effect.

In an attempt to fill this research gap, we examined the effectiveness of a CBT in reducing socially anxious classroom behavior in students with severe symptoms of social anxiety. In addition to the question of the extent to which the problematic behavior can be reduced during the intervention (research question 1), we are particularly interested in the extent to 
which individual differences exist in the responsiveness to the training between the students and which patterns of effectiveness will emerge (research question 2). We hypothesize that CBT will reduce socially anxious behavior with a moderate to strong effect, but that there will be differences between the students in regard to which specific behaviors are improved and whether the intervention effect occurs immediately after or continuously during the implementation of the training.

\section{MATERIALS AND METHODS}

\subsection{Participants}

This study was conducted with a single class of third-graders of an inclusive primary school in North-Rhine Westphalia (Western Germany). This class was selectively chosen, since the class teacher reported major concerns to the second author regarding her students' internalizing classroom behaviors. The age group was selected because children in the third grade have already been in school for two years and possible adjustment effects on internalizing instructional behavior are less likely than in the first two years of school attendance. The participating students were selected based on three criteria: First, the participating students should have severe symptoms of social anxiety; second, these symptoms should manifest in a similar manner, so that the interventions responses of the students would be comparable across multiple baselines; third, to control for potential gender effects on the students' responses to the intervention, students should be variant with respect to gender so that, at best, both girls and boys participate in the study.

In a first step, the classroom teacher completed the problem scales anxious/depressive (AD) and withdrawal/depressive (WD) of the German language version of the Teacher Report Form from the Child Behavior Checklist (TRF; Döpfner et al., 2015). The results of these scales indicate whether the students exhibit internalizing problem behaviors associated with social anxiety. In a second steps, the students who showed significant behavior problems in both TRF scales (T-score $\geq 60$ ) were compared in regard to their age and their gender with the aim of ensuring comparability across baselines. The AD scale consists of 16 items that are rated by the teacher on a scale from 0 (not true) to 2 (very or often true). The scale can thus take on a raw score of $0-32$, whereby for boys and girls aged up to a maximum of eleven years, a score of 6 already indicates a behavioral problem (T-value $>60$ ). The WD scale consists of eight items assessed on the same scale. Accordingly, the scale can take a raw score from 0 to 16 , whereby a score of 3 already indicates a behavior problem ( $\mathrm{T}$-value $>60$ ). Following this procedure, it was found that two female and two male students exhibited very high levels of socially anxious behavior: Liam (9-years-old; T-scores for $\mathrm{AD}=71$, and $\mathrm{WD}=$ 81 ), Janet (10-years-old; $\mathrm{T}$-scores for $\mathrm{AD}=69$, and $\mathrm{WD}=81$ ), Emma (9-years-old; $\mathrm{T}$-scores for $\mathrm{AD}=71$, and $\mathrm{WD}=85$ ), and Noah (10-years-old; $\mathrm{T}$-scores for $\mathrm{AD}=76$, and $\mathrm{WD}=85$ ). All four students had comparable T-scores in both scales of the TRF, and they all experienced slightly more problems regarding the issues assessed with the scale WD. Since the four students met the aforementioned criteria, all of them participated in the study.
Before the study began, the students and their parents were provided with detailed descriptions of the study's purpose and procedures, and potential questions were answered. Furthermore, the parents were informed about the use of the data and the associated right of withdrawal.

\subsection{Measures}

\subsubsection{Child Behavior Checklist-Teacher Report Form} The Child Behavior Checklist-Teacher Report Form (CBCLTRF) (Döpfner et al., 2015) is a rating scale to be completed by the teacher that focuses on clinically relevant problem behaviors. The form consists of 120 items focusing on child externalizing and internalizing behavioral problems as well as somatic problems. Ratings are made on a scale of 0 (not true) to 2 (very true or often true). In our study, the empirically based syndrome scales Anxious/Depressive (AD) and Withdrawal/ Depressive (WD) of the TRF were selected based on the degree to which they were considered to measure behavioral problems associated to social anxiety. Studies examining the psychometric properties of the German version of the TRF suggest good internal consistencies for those scales (Döpfner et al., 2015). The usage of the TRF in this study were twofold: First, it was used to identify students suitable to participate in the study, and, second, it served as a pre-post criterion measure before and after the intervention.

\subsubsection{Integrated Teacher Report Form}

The Integrated Teacher Report Form (ITRF) was developed initially to assess externalizing and internalizing classroom behaviors indicating a social, emotional, and behavioral risk in students (Volpe et al., 2018; Volpe et al., 2020). It consists of 36 items measuring academic productivity problems (eight items), oppositional/disruptive behavior (eight items), anxious/ depressive behavior (eleven items), and social withdrawal (nine items). The ITRF is part of the Integrated Screening and Intervention System (Volpe and Fabiano, 2013), which incorporates universal screening, intervention, and behavioral progress monitoring. Numerous studies support its factorial validity, internal consistency and retest reliability (Daniels et al., 2014; Volpe et al., 2018; Volpe et al., 2020), the construct validity (Casale et al., 2019), and the cross-cultural equivalence (Casale et al., 2018). In this study, we used the internalizing behavior scales of the ITRF (anxious/depressive, social withdrawal) to precisely identify the problematic classroom behaviors raising the most concern for the students. The teacher completed the ITRF items on a 4 -point Likert scale $(0=$ behavior is not of concern, 1 = behavior is of slight concern, 2 = behavior is of moderate concern, 3 = behavior is of strong concern).

\subsubsection{Direct Behavior Rating-Multiple Item Scales}

Direct Behavior Rating-Multiple Item Scales (DBR-MIS; Christ et al., 2009) were used in the present study to record the trajectories of the socially anxious classroom behaviors. DBRMIS is a combination of systematic direct observation and behavior rating scales in order to provide a psychometrically defensible, but cost- and time-efficient tool (Christ et al., 2009). DBR-MIS is used by concretely operationalizing a student's 
behavior (e.g., concentrated work) and observing this behavior in a concrete situation of relevance (e.g., periods of silent work). DBR-MIS usually define 2 to 5 items referring to specific and concrete student behaviors, which are rated on a Likert scale immediately after the target situation. This procedure is efficient and flexible, and therefore well-suited for measuring behavioral progress within quantitative single-case studies (see e.g., Matta et al., 2020). Numerous studies indicate good to excellent accuracy, reliability, validity, and sensitivity to change of DBRMIS (see e.g., Casale et al., 2021; Chafouleas et al., 2012; Matta et al., 2020; Smith et al., 2018; Volpe and Briesch, 2015). The psychometric quality of DBR could also be demonstrated for the assessment of internalizing behavior, i.e., depressive behaviors (Kilgus et al., 2019) and academic anxiety (van der Embse et al., 2015).

To identify relevant items for the DBR-MIS, a step-by-step approach following Hyman et al. (1998) was chosen. First, the most problematic internalizing classroom behaviors exhibited by the four students were identified using the results of the ITRF. In the second step, interviews were conducted with the class teacher to verify whether these items were generally observable for the respective students in class. On this basis, the following four items related to social anxiety were retained: 1) Avoids social interactions, 2) Prefers to play alone, 3) Acts overly shy, and 4) Does not participate in class. These items have acceptable loadings of $\beta=0.49$ to 0.83 on the socially withdrawn behavior factor of the ITRF $(\alpha=0.88, \omega=0.90)$ and thus adequately represent a key feature of socially anxious symptom behavior (Volpe et al., 2020). The occurrence of these behaviors across the day were rated at the end of each day on four days per week during the baseline (twelve measurement points) and intervention (36 measurement points) phases. The items were rated on a four-point Likert scale $(0=$ behavior did not occur, $1=$ behavior occurred slightly, 2 = behavior occurred often, 3 = behavior occurred almost always).

\subsection{Procedures and Study Design}

After the identification of the students to participate in the study, the class teacher completed the ITRF to precisely operationalize the students' internalizing behavior specifically in classroom settings. Based on the results of the ITRF, the aforementioned procedure was conducted to create the DBR-MIS to measure the students' progress in their socially anxious classroom behavior. The baseline started in January 2018, immediately after the Christmas break in Germany, and lasted for three weeks. The four problematic behaviors were rated on four school days a week at the end of each school day for each participating student. This was to ensure that the DBR results represented the socially anxious classroom behavior across the entire school day. In sum, each student's behavior was rated on twelve measurement points in the baseline.

This was followed by the intervention phase after three weeks. The intervention was carried out using the "Mutig werden mit Til Tiger" program (English translation: "Becoming brave with Til the Tiger"; Ahrens-Eipper et al., 2010), which is a manualized cognitive-behavioral training program for children aged five to ten. The training consists of nine group sessions, in which students learn and practice confident behavior in social situations. The overarching goals of the training are to reduce social avoidance and to build up socially competent behaviors. The main character accompanying the children through the training is the hand puppet Til, a shy tiger who does not dare to do many things, but then learns and tries out new things together with the children. The intervention session follows a certain routine, or ritual, which makes them manageable, and familiar for the participants. Each session starts with a reflection on the past week in which students report positive and negative experiences. This is followed by a discussion of a self-observation sheet, where the children note whether they have achieved the given weekly goal (e.g., participating in group activities, talking in front of the class) on a daily basis by coloring a tiger. Afterwards, each session has a central topic, which is introduced through a game or an experience of the tiger. Following this, the topic is made tangible for the students through a role play and practiced accordingly. This is followed by a short form of progressive muscle relaxation. At the end of each lesson, the students receive a new self-observation sheet as homework.

In our study, the intervention was carried out by a special education teacher in training who led the sessions (i.e., the second author). This person had previously been trained in both the assessment of internalizing behavior in the classroom and the implementation of CBT in a 3-months university course. The intervention was provided across nine weeks (one session per week), whereby the starting point of the intervention could not be staggered due to the fact that the intervention was a grouptraining in only one class. If a student was absent due to illness or other school activities, the session was rescheduled to another day in the week, which ensured that the intervention could be implemented in its entirety for all participants. Rating of the socially anxious behaviors in the intervention phase was made four times a week by the aforementioned special education teacher in training, yielding a total of 36 measurement points in the intervention phase.

\subsection{Analyses}

The main dependent variable in our data analyses were the DBRMIS scores. We conducted the data analyses with both the individual score of each single behavior item on the DBR-MIS and the sum score of all four items of the DBR-MIS. Therefore, five dependent variables were used for the data analyses per measurement point: four single behavior scores, each with values from 0 to 3, and the DBR-MIS sum score with values between 0 and 12 . This procedure was chosen to be able to make differential inferences regarding the students' individual behavioral responses to the intervention.

To examine the intervention effects on the socially anxious classroom behavior of each student, several procedures were used to analyze the data, including descriptive statistics, and inferential statistics based on regression analysis. First, we calculated descriptive statistics (mean values and standard deviations for baseline and intervention), and calculated the non-rescaled non-overlap of all pairs (NAP; Parker and 
Vannest, 2009), and Tau-U (Parker et al., 2011). The NAP is the percentage of all pairwise comparisons across the baseline and treatment phases. According to Parker and Vannest (2009), medium effects are indicated by values of $66-92 \%$, and strong effects are indicated by values of $93-100 \%$. Tau-U analysis represent an analysis technique that allows to examine treatment effects on both between-phase difference and within-phase trend (Parker et al., 2011), and which offers at least four different types of Tau-U calculation (Parker et al., 2011). In this study, we used the Tau-U "non-overlap with Phase B trend with baseline trend controlled" (Parker et al., 2011, p. 291), which is the non-overlap of all pairs between the baseline and intervention phase plus the intervention phase trend minus the baseline phase trend. This, then, allows us to describe quantitatively how many percent of phase B scores have decreased over all phase A scores while the experimental treatment did not appear to be affected by potential trend of scores (Brossart et al., 2018). Although no general recommendation can be made about conventions for interpreting Tau-U values, we considered a value of 0.20 as a small change, values from 0.20 to 0.60 as moderate changes, values from 0.60 to 0.80 as large changes, and values above 0.80 as large to very large changes as a guideline for interpreting the results (Vannest and Ninci, 2015).

After arriving at the results of the calculations described, we analyzed the data using a piecewise-regression approach (Huitema and McKean, 2000). This procedure enables us to control developmental trends in the data (trend effects) and differentiate between continuous (slope effect) and immediate (level effects) intervention effects. We conducted several piecewise regression models for each single case (for each single behavior, and for the sum score of the DBR-MIS) and a multilevel extension across all cases (again for each single behavior, and for the sum score of the DBR-MIS) with measurements at level 1 nested in subjects at level 2 (Van den Noortgate and Onghena, 2003). These combination of regression models allow for inferences about a) the interventions effects for each single case differentiated for each individual behavior as well as for the overarching behavioral dimensions of socially anxious behavior in the classroom, and b) the intervention effects across all four students, also differentiated by individual behaviors as well as the higher-level behavioral dimension of social anxiety.

Finally, we calculated the Reliable Change Index (RCI) for the difference between the TRF pre- and post-scores for the AD and WD scales for each individual student. Basically, the RCI represents the extent to which the magnitude of behavioral change is statistically reliable (Jacobson and Truax, 1992). We calculated the RCI based on the following formula (Jacobson and Truax, 1992):

$$
R C I=\frac{X_{\text {post }}-X_{\text {pre }}}{\sqrt{2\left(S_{\text {pre }} \sqrt{1-R_{x x}^{2}}\right)}}
$$

where $X_{\text {post }}$ and $X_{\text {pre }}$ represent the TRF pre- and post-scores for each of the participating students, $S_{\text {pre }}$ is the standard deviation of the norm data, and $\mathrm{R}_{\mathrm{xx}}$ indicates the test-retest reliability of the TRF scales. If the resulting score has a value above 1.96, the TRF score difference between pre- and post-test is statistically significant and indicates a statistically reliable change of the TRF scores before and after the intervention.

All descriptive and single-case analyses were conducted using R (R Core Team, 2018) and the package scan (Wilbert and Lüke, 2019). The reliable change indices were calculated in Microsoft Excel.

\section{RESULTS}

\subsection{Overlap Effect Sizes}

\subsubsection{Liam}

Descriptive results and overlap effect sizes are presented in Table 1 and Table 2. The graphs of the behavioral trajectories of all students are shown in Figure 1. Both NAP (92.59\%) and Tau-U (-0.81) of Liam's sum score on socially anxious behavior indicate a strong reduction in the overall problematic behavior in the intervention phase. Looking at the individual behaviors, both the NAP and the Tau-U indicate moderate to strong effects, with the largest effects in the item "Does not participate in class."

\subsubsection{Janet}

For Janet, a strong intervention effect can be found across the sum of all socially anxious classroom behaviors in both the NAP (94.10\%) and the Tau-U (-0.83). Similar to Liam, NAP and Tau$\mathrm{U}$ indicate moderate to strong effects for each individual behavior, with the largest effects in the item "Does not participate in class."

\subsubsection{Emma}

Emma's socially anxious behavior was reduced with a medium effect in the intervention phase according to NAP (79.40\%) and with a large effect in the intervention phase according to Tau-U $(-0.75)$. Looking at the individual behaviors, Emma shows a smaller reduction in problem behaviors overall, although NAP and Tau-U still indicate at least moderate effects. Emma shows the strongest behavioral changes in the item "Prefers to play alone."

\subsubsection{Noah}

In Noah's sum score, the NAP (89.47\%) indicates a moderate effect and the Tau-U (-0.78) indicates a large effect. As with all other three students, the NAP and Tau-U values for the individual behaviors indicate moderate to strong effects. Noah shows the strongest reduction in the problem behavior "Acts overly shy."

\subsection{Piecewise Regression Models \\ 4.2.1 Liam}

The results of the piecewise regression models are presented in Table 3. When summed across all behaviors, Liam's progress shows a significant trend effect in the A phase. This means that 
TABLE 1 | Descriptive Statistics and Overlap Indices for the Single Behavior Scores and the DBR-MIS Sum Scores for each Student.

\begin{tabular}{|c|c|c|c|c|c|c|c|c|c|c|}
\hline & \multirow[t]{2}{*}{$\mathrm{n}_{\mathrm{A}}$} & \multirow[t]{2}{*}{$\mathrm{n}_{\mathrm{B}}$} & \multicolumn{6}{|c|}{ Descriptive statistics } & \multicolumn{2}{|c|}{ Overlap Indices } \\
\hline & & & $M_{A}$ (SD) & $M_{B}$ (SD) & $\mathbf{M e d}_{A}$ & $\operatorname{Med}_{B}$ & Trend $_{A}$ & TrendB & NAP & Tau-U (p) \\
\hline \multicolumn{11}{|l|}{ Liam } \\
\hline Item 2 & 12 & 36 & $1.92(0.51)$ & $1.28(0.61)$ & 2.00 & 1.00 & -0.08 & -0.04 & 75.81 & $-0.75(<0.000)$ \\
\hline Item 3 & 12 & 36 & $1.92(0.51)$ & $1.44(0.56)$ & 2.00 & 1.00 & -0.10 & -0.04 & 70.25 & $-0.80(<0.000)$ \\
\hline Item 4 & 12 & 36 & $2.42(0.51)$ & $1.14(0.72)$ & 2.00 & 1.00 & 0.01 & -0.04 & 90.28 & $-0.79(<0.000)$ \\
\hline \multicolumn{11}{|l|}{ Janet } \\
\hline Item 1 & 12 & 36 & $2.08(0.67)$ & $1.42(0.50)$ & 2.00 & 1.00 & -0.12 & -0.04 & 76.04 & $-0.83(<0.000)$ \\
\hline Item 2 & 12 & 36 & $2.08(0.29)$ & $1.50(0.65)$ & 2.00 & 1.00 & 0.03 & -0.03 & 75.58 & $-0.70(<0.000)$ \\
\hline Item 3 & 12 & 36 & $2.08(0.51)$ & $1.44(0.50)$ & 2.00 & 1.00 & -0.06 & -0.03 & 77.31 & $-0.79(<0.000)$ \\
\hline Item 4 & 12 & 36 & $2.75(0.45)$ & $1.33(0.63)$ & 3.00 & 1.00 & 0.00 & -0.03 & 94.79 & $-0.77(<0.000)$ \\
\hline Sum Score & 12 & 36 & $9.00(1.28)$ & $5.69(1.58)$ & 9.00 & 5.00 & -0.14 & -0.13 & 94.10 & $-0.83(<0.000)$ \\
\hline \multicolumn{11}{|l|}{ Emma } \\
\hline Item 4 & 12 & 36 & $2.25(0.45)$ & $1.72(0.70)$ & 2.00 & 2.00 & 0.02 & -0.05 & 70.49 & $-0.76(<0.000)$ \\
\hline Sum Score & 12 & 36 & $9.25(1.14)$ & $7.17(2.04)$ & 9.50 & 7.00 & -0.17 & -0.18 & 79.40 & $-0.75(<0.000)$ \\
\hline \multicolumn{11}{|l|}{ Noah } \\
\hline Item 1 & 12 & 36 & $2.58(0.51)$ & $2.14(0.64)$ & 3.00 & 2.00 & -0.05 & -0.03 & 68.17 & $-0.67(<0.000)$ \\
\hline Item 2 & 12 & 36 & $2.67(0.49)$ & $1.86(0.59)$ & 3.00 & 2.00 & 0.05 & -0.02 & 81.94 & $-0.74(<0.000)$ \\
\hline Item 3 & 12 & 36 & $2.67(0.65)$ & $1.89(0.46)$ & 3.00 & 2.00 & 0.05 & -0.03 & 82.87 & $-0.84(<0.000)$ \\
\hline Item 4 & 12 & 36 & $2.75(0.45)$ & $2.06(0.67)$ & 3.00 & 2.00 & 0.00 & -0.05 & 77.43 & $-0.78(<0.000)$ \\
\hline Sum Score & 12 & 36 & $10.58(1.16)$ & 7.97 (1.59) & 11.00 & 8.00 & 0.00 & -0.13 & 89.47 & $-0.78(<0.000)$ \\
\hline
\end{tabular}

Note. Item 1 = Avoids Social Interactions, Item 2 = Prefers to Play Alone, Item $3=$ Acts Overly Shy, Item $4=$ Does Not Participate in Class. The sum scores for each student is presented in bold letters.

there was already a reduction in problem behavior in the A phase. The individual behaviors "Prefers to play alone" and "Acts overly shy" also show a significant trend in the A phase. The problem behavior "Acts overly shy" also shows a significant level effect, which indicates that a sudden improvement in behavior occurred as the implementation of the intervention began.

\subsubsection{Janet}

No significant effects can be found in Janet's sum score. The analysis of the individual behaviors shows, however, that significant trend, level, and slope effects can be found in the behavior "Avoids social interactions." This means that Janet's tendency to avoid social interaction already decreased in the A phase, but could be reduced immediately with the implementation of the intervention and additionally decreased significantly by 0.08 scale points per measurement point during the intervention phase. In addition, the significant level effect in the behavior "Does not participate in class" indicates a sudden improvement in class participation immediately after the implementation of the intervention.

\subsubsection{Emma}

A significant trend effect in the A phase can also be observed for Emma in the sum score. In addition, however, there is also quite a strong and significant level effect, which indicates a sudden improvement in behavior once the intervention began. The data also show significant trend, level, and slope effects for Emma in the item "Prefers to play alone," which, in addition to the reduction of playing alone already found in the A phase, also indicate a sudden reduction after the implementation of the intervention as well as a continuous improvement by 0.09 scale points per measurement time point in the $\mathrm{B}$ phase.

\subsubsection{Noah}

The only effect found for Noah was a significant slope effect in the item "Acts overly shy," indicating a progressively decreasing shy behavior during the intervention phase of 0.08 scale points per measurement.

\subsection{Hierarchical Piecewise Regression}

Multilevel analysis of the aggregated four individual cases as a single case with measurement points (Level 1) clustered into students (Level 2) shows a significant trend effect in the A phase for the overall socially anxious behavior as well as a significant level effect (see Table 4 for all results). The level effect indicates an improvement in behavior averaging at 0.59 scale point that occurred immediately after the implementation of the intervention. A significant level effect can also be found for the item "Avoids social interactions," for which the behavioral 
TABLE 2 | Descriptive Statistics and Overlap Indices for the DBR-MIS Sum Scores for each Student.

\begin{tabular}{|c|c|c|c|c|c|c|c|c|c|c|}
\hline \multirow[t]{2}{*}{ Student } & \multirow[t]{2}{*}{$n_{\mathrm{A}}$} & \multirow[t]{2}{*}{$n_{B}$} & \multicolumn{6}{|c|}{ Descriptive statistics } & \multicolumn{2}{|c|}{ Overlap Indices } \\
\hline & & & $M_{A}(S D)$ & $M_{B}$ (SD) & $\operatorname{Med}_{A}$ & $\operatorname{Med}_{B}$ & Trend $_{A}$ & TrendB & NAP & Tau-U (p) \\
\hline Liam & 12 & 36 & $8.50(1.24)$ & $5.47(1.68)$ & 9.00 & 6.00 & -0.15 & -0.15 & 92.59 & $-0.81(<0.000)$ \\
\hline Janet & 12 & 36 & $9.00(1.28)$ & $5.69(1.58)$ & 9.00 & 5.00 & -0.14 & -0.13 & 94.10 & $-0.83(<0.000)$ \\
\hline Emma & 12 & 36 & $9.25(1.14)$ & $7.17(2.04)$ & 9.50 & 7.00 & -0.17 & -0.18 & 79.40 & $-0.75(<0.000)$ \\
\hline
\end{tabular}
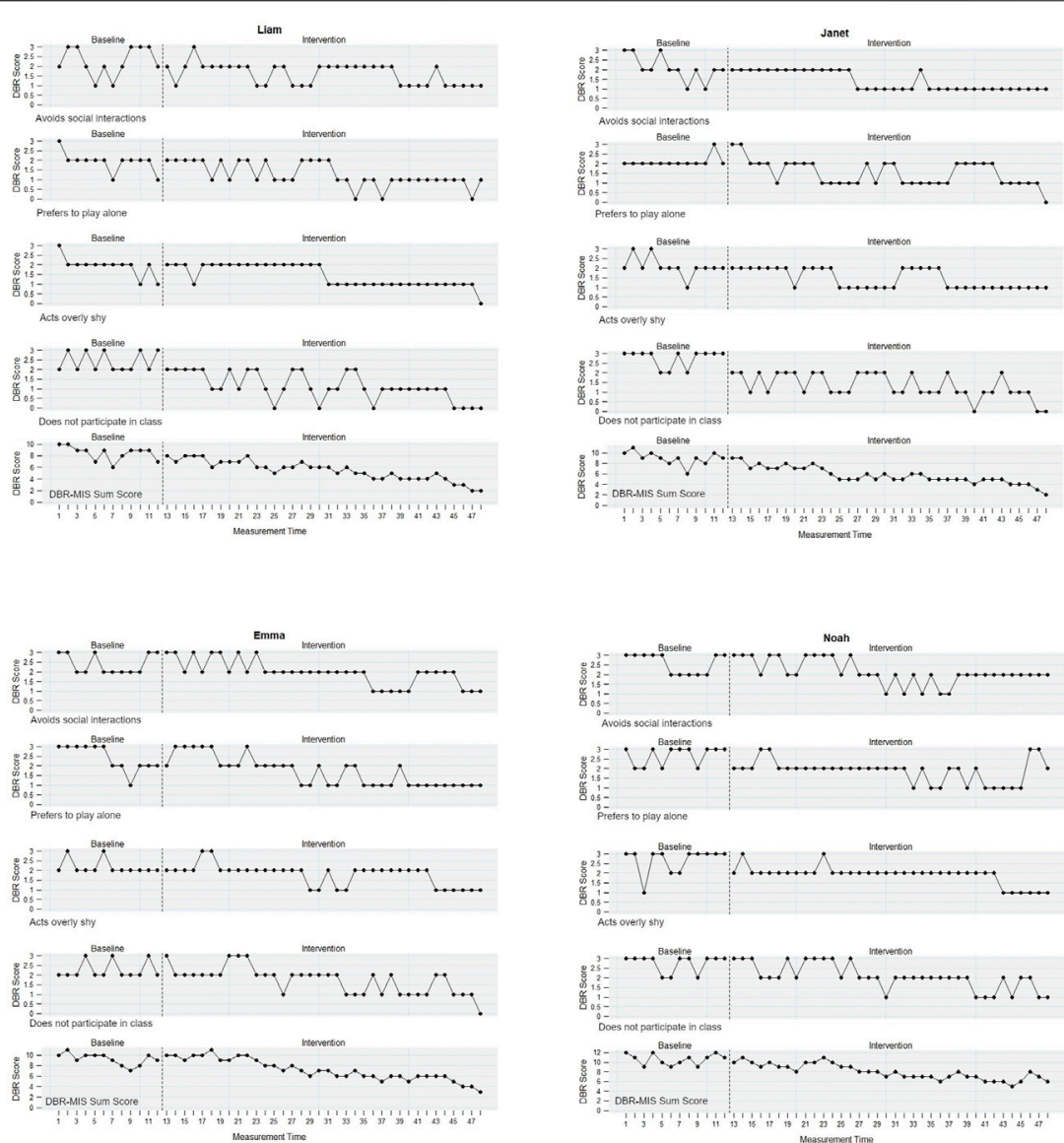

FIGURE 1 | Trajectories of the socially anxious classroom behavior for all four students during A- and B-phase.

improvement that occurred with the implementation of the intervention can be quantified with 0.32 scale points across all four students. The significant slope effect observed for the item "Does not participate in class" shows that there was a continuous improvement in class participation across all four students during the intervention phase, indexed with 0.05 scale points per measurement.

\subsection{Reliable Change Index}

The analysis of the pre- and post-scores of the AD scale from the TRF shows that all four students are still in the problematic
T-score range of $\mathrm{T}>60$ after the intervention (see Table 5 for all results). Nevertheless, the RCI indicates a statistically reliable reduction in raw scores with strong effects for all four students.

A similar pattern can be observed for the WD scale. Here, too, all four students are still in the problematic T-score range after the intervention, though the RCI does show statistically reliable raw score improvements with strong effects for Liam, Janet, and Emma. For Noah, the RCI is only slightly below the cut-off for statistically reliable changes in the $95 \%$ confidence interval. 
TABLE 3 | Piecewise regression analyses for the single behavior scores and the DBR-MIS sum scores for each student.

\begin{tabular}{|c|c|c|c|c|}
\hline & Intercept (SE) & Trend & Level & Slope \\
\hline \multicolumn{5}{|l|}{ Liam } \\
\hline Item 1 & $2.09^{\star \star}(0.35)$ & 0.02 & -0.33 & -0.05 \\
\hline Item 2 & $2.44^{\star \star}(0.29)$ & $-0.08^{\star}$ & 0.49 & 0.04 \\
\hline Item 3 & $2.58^{\star \star}(0.21)$ & $-0.10^{\star \star}$ & $-0.87^{\star \star}$ & 0.06 \\
\hline Item 4 & $2.35^{\star \star}(0.35)$ & 0.01 & -0.54 & -0.05 \\
\hline Sum Score & $9.46^{\star \star}(0.50)$ & $-0.15^{\star}$ & 0.50 & 0.00 \\
\hline \multicolumn{5}{|l|}{ Janet } \\
\hline Item 1 & $2.83^{\star \star}(0.23)$ & $-0.12^{\star \star}$ & $-0.67^{\star \star}$ & $-0.08^{\star}$ \\
\hline Item 2 & $1.88^{\star \star}(0.32)$ & 0.03 & -0.17 & -0.06 \\
\hline Item 3 & $2.47^{\star \star}(0.26)$ & -0.06 & 0.24 & 0.03 \\
\hline Item 4 & $2.73^{\star \star}(0.32)$ & 0.00 & $-0.82^{\star}$ & -0.04 \\
\hline Sum Score & $9.91^{\star \star}(0.54)$ & -0.14 & -0.06 & 0.01 \\
\hline \multicolumn{5}{|l|}{ Emma } \\
\hline Item 1 & $2.49^{\star \star}(0.29)$ & -0.01 & 0.45 & -0.04 \\
\hline Item 2 & $3.35^{\star \star}(0.27)$ & $-0.14^{\star \star}$ & $-1.14^{\star \star}$ & $-0.09^{\star}$ \\
\hline Item 3 & $2.39^{\star \star}(0.27)$ & -0.04 & 0.37 & 0.01 \\
\hline Item 4 & $2.14^{\star \star}(0.31)$ & 0.02 & 0.23 & -0.06 \\
\hline Sum Score & $10.36^{\star *}(0.50)$ & $-0.17^{\star}$ & $-2.12^{\star \star}$ & -0.01 \\
\hline \multicolumn{5}{|l|}{ Noah } \\
\hline Item 1 & $2.92^{* \star}(0.33)$ & -0.05 & 0.46 & 0.02 \\
\hline Item 2 & $2.35^{\star \star}(0.34)$ & 0.05 & -0.70 & -0.07 \\
\hline Item 3 & $2.35^{\star \star}(0.27)$ & 0.05 & -0.50 & $-0.08^{*}$ \\
\hline Item 4 & $2.77^{\star \star}(0.30)$ & 0.00 & 0.16 & -0.04 \\
\hline Sum Score & $10.56^{\star \star}(0.58)$ & 0.00 & -0.25 & -0.13 \\
\hline
\end{tabular}

Note. ${ }^{*}=$ significant $(\mathrm{p}<0.05) ;{ }^{* *}=$ significant $(\mathrm{p}<0.001)$. Item $1=$ Avoids Social Interactions, Item 2 = Prefers to Play Alone, Item $3=$ Acts Overly Shy, Item $4=$ Does Not Participate in Class. The sum scores for each student is presented in bold letters.

\section{DISCUSSION}

\subsection{Main Findings}

The purpose of this study was to analyze the effects of a cognitivebehavioral intervention in a school setting on socially anxious classroom behavior in primary school students. In addition to descriptive and visual analysis, as well as an analysis of overlapping data points from phases $\mathrm{A}$ and $\mathrm{B}$, we were particularly interested in the differential patterns of effects, which we analyzed using regression models.

Overall, descriptive and visual analysis, as well as overlap indices, indicate moderate to large reductions in socially anxious behaviors in the classroom for all participating students during the intervention phase. The clinical significance of the effects is further supported by substantially lower raw scores in the AD and WD problem scales of the TRF, which are significant and reliable according to the RCI values. These results indicate that all four students benefited from the intervention and that socially anxious behaviors decreased in the course of the intervention with moderate to strong effects. The existing trend effects in the baseline phase could be due to the start of the survey immediately after the school vacations, after which students with internalizing behavior problems may often be more withdrawn and shyer. However, the analysis procedures used here take these trend effects into account. This study was thus able to replicate the positive effects of CBT on socially anxious behavior found in group studies (Scaini et al., 2016). These positive effects can also be supported, in particular, in a complementary way by the fact that socially anxious behaviors persist in a very stable manner, especially among children of primary school age (e.g., Broeren et al., 2013).

The specific nature and extent of this positive response of the four students to the CBT intervention, however, did show some differences. While Liam and Janet improved primarily in their participation in class, Emma showed the greatest improvement in playing with others and Noah in becoming less shy. This confirms that the change sensitivity of higher-level behavioral dimensions is always measured by particularly salient specific behavioral dimensions, and accordingly, an appropriate level of specificity of the promoted behaviors should be ensured (Volpe and Briesch, 2015).

This impression is also confirmed by the results of the piecewise regression analyses. Here, most effects can be found for the individual behaviors-and not for the sum score of the DBR-MIS. Interestingly, depending on the student and the behavior being examined, both level effects and slope effects can be found. This finding is also supported by the multilevel regressions across all measurement points of the four students together. Here, level effects can be found for the sum of all socially anxious behaviors and socially avoidant behaviors as well as a slope effect for participation in class. Level effects indicate a sudden change in behavior that occurred

TABLE 4 | Multilevel piecewise-regression models for all behavior dimensions.

\begin{tabular}{|c|c|c|c|c|}
\hline Parameter & B & SE & $t$ & $p$ \\
\hline \multicolumn{5}{|c|}{ Avoids Social Interactions } \\
\hline Intercept & 2.58 & 0.20 & 12.99 & $<0.001^{\star \star}$ \\
\hline Trend & -0.04 & 0.02 & -1.85 & 0.066 \\
\hline Level Phase B & -0.32 & 0.16 & 1.99 & $0.049^{\star}$ \\
\hline Slope Phase B & 0.00 & 0.02 & 0.15 & 0.884 \\
\hline \multicolumn{5}{|c|}{ Prefers to Play Alone } \\
\hline Intercept & 2.50 & 0.20 & 12.77 & $<0.001^{\star *}$ \\
\hline Trend & -0.04 & 0.02 & -1.64 & 0.102 \\
\hline Level Phase B & 0.19 & 0.17 & 1.14 & 0.256 \\
\hline Slope Phase B & -0.00 & 0.02 & --0.04 & 0.965 \\
\hline \multicolumn{5}{|l|}{ Acts Overly Shy } \\
\hline Intercept & 2.45 & 0.17 & 14.78 & $<0.001^{\star \star}$ \\
\hline Trend & -0.04 & 0.02 & -2.08 & $0.039^{*}$ \\
\hline Level Phase B & 0.24 & 0.14 & 1.80 & 0.074 \\
\hline Slope Phase B & 0.00 & 0.02 & 0.21 & 0.837 \\
\hline \multicolumn{5}{|c|}{ Does not Participate in Class } \\
\hline Intercept & 2.50 & 0.12 & 11.66 & $<0.001^{\star \star}$ \\
\hline Trend & 0.01 & 0.02 & 0.31 & 0.755 \\
\hline Level Phase B & -0.24 & 0.17 & -1.40 & 0.162 \\
\hline Slope Phase B & -0.05 & 0.02 & -2.15 & $0.033^{*}$ \\
\hline \multicolumn{5}{|c|}{ Socially Anxious Classroom Behavior (Sum Score) } \\
\hline Intercept & 10.07 & 0.55 & 18.23 & $<0.001^{\star \star}$ \\
\hline Trend & -0.11 & 0.04 & -3.03 & $0.003^{\star \star}$ \\
\hline Level Phase B & -0.59 & 0.29 & 2.06 & $0.041^{*}$ \\
\hline Slope Phase B & -0.03 & 0.04 & -0.88 & 0.379 \\
\hline
\end{tabular}

Note. ${ }^{*}=$ significant $(\mathrm{p}<0.05) ;{ }^{* *}=$ significant $(\mathrm{p}<0.001)$. 
TABLE 5 | Reliable chance index for pre- posttest differences for anxious/depressive behavior and withdrawal/depressive behavior

\begin{tabular}{|c|c|c|c|c|c|c|c|c|c|c|c|c|}
\hline \multirow[b]{3}{*}{ Student } & \multicolumn{6}{|c|}{ Anxious/Depressive } & \multicolumn{6}{|c|}{ Withdrawal/Depressive } \\
\hline & \multicolumn{2}{|c|}{ Pretest } & \multicolumn{4}{|c|}{ Posttest } & \multicolumn{2}{|c|}{ Pretest } & \multicolumn{4}{|c|}{ Posttest } \\
\hline & RS & $\mathbf{T}$ & RS & $\mathbf{T}$ & $d$ & $\mathrm{RCI}^{\mathrm{a}}$ & RS & $\mathbf{T}$ & RS & $\mathbf{T}$ & d & $\mathrm{RCl}^{\mathrm{a}}$ \\
\hline Liam & 12 & 71 & 8 & 66 & -1.79 & $-2.82^{\star}$ & 12 & 71 & 6 & 66 & -2.00 & $-3.16^{\star}$ \\
\hline Janet & 12 & 69 & 9 & 65 & -1.34 & $-2.12^{\star}$ & 12 & 69 & 6 & 66 & -2.00 & $-3.16^{\star}$ \\
\hline Emma & 14 & 71 & 10 & 67 & -1.79 & $-2.82^{\star}$ & 14 & 71 & 7 & 68 & -2.00 & $-3.16^{\star}$ \\
\hline Noah & 15 & 76 & 12 & 71 & -1.34 & $-2.12^{\star}$ & 15 & 76 & 9 & 73 & -1.2 & -1.90 \\
\hline
\end{tabular}

Note. RS, raw score, $T=T$-score

${ }^{a}$ Values above + -1.96 indicate a statistically reliable change of the scores before and after the intervention (Jacobson \& Truax, 1992).

immediately after the implementation of the intervention, whereas slope effects indicate a continuous and progressive change in behavior (Rindskopf and Ferron, 2014). Because the cognitive-behavioral intervention implemented here targeted both cognition-related and behavioral-social skills, this could mean that, in addition to a general responsiveness of the students to a particular method, there could also be an interaction between responsiveness, the individual expression of the students' social anxiety, and individual intervention elements. This in turn can also cause the intervention effect to be more progressive (as in a significant level effect) or more progressively (as in a significant slope effect) evident in concrete behavior. Such differential moderation effects could be explained by integrative models of self-regulated behavior (e.g., Bailey and Jones, 2019), in which generic cognitive, emotional, and social self-regulated skills are linked with executive functions and conscious, intentional cognitive-behavioral control. Initial experimental and intervention studies in children and adolescents with social anxiety have already empirically demonstrated the existence of these mechanisms (see e.g., Pergamin-Hight et al., 2016; Abend et al., 2018).

\subsection{Limitations}

The $\mathrm{AB}$ design implemented here is a quasi-experimental singlecase study design (Barlow et al., 2009). Due to the fact that this design consists solely of $1 \mathrm{~A}$ and $1 \mathrm{~B}$ phase and neither phase is implemented reversely (such as in an $\mathrm{ABA}$ or an $\mathrm{ABAB}$ design), the internal validity of the study is limited in that potential maturation and history effects were not controlled for (Tate et al., 2016). Therefore, a causal statement that the effects found were due to the intervention is not valid. Nevertheless, the design can be used to show whether a meaningful change in behavior occurred during the intervention, which is the most important information from a practical perspective (Giannakakos and Lanovaz, 2019).

Another limitation of this study is that both clinically relevant symptom behavior identified using the TRF (in the teacher's judgment) and socially anxious behavior in the classroom (in the teacher's judgment) were collected exclusively by teacher (in training) ratings. This is problematic primarily for the reason that internalizing behavioral problems are generally more difficult for other people to observe than, for example, externalizing behavior problems, and that there are substantial multi-informant effects in the assessment of internalizing behavioral problems (See e.g., De Los Reyes et al., 2015; Huber et al., 2019). Therefore, our data could be biased by an underestimation of the frequency of occurrence of the problem behaviors. On the other hand, it has been shown that teachers can identify internalizing behavior problems quite accurately if they are trained in advance on how to describe them (Conley et al., 2014).

Another limitation of this study-as with all single case studies - is the generalizability of the results. The effects of CBT on socially anxious behavior found in this study for four students do not allow for a conclusion that CBT can reduce socially anxious behavior for the population of all primary school students with social anxiety symptoms (Maggin et al., 2018). Rather, the generalizability of controlled individual case studies is established by their replication. Only through a larger number of controlled single-case studies, whose findings are statistically integrated in a meta-analysis, would any inferences about a target population be permissible.

\subsection{Implications and Future Research}

The present study contributes to the existing body of research on the effectiveness of CBT in the school context and extends it by a) for the first time, evaluating a controlled single-case study in a differentiated manner using regression models and b) not analyzing clinically relevant symptom behavior, but actual socially anxious behavior occurring in the classroom. The positive findings underscore the usefulness of using CBT in schools for supporting students with social anxiety (Scaini et al., 2016) and illustrate that each student responds differently and individually to interventions. Further singlecase studies, at best with experimental designs, are needed to reliably generalize the effects to other populations (Tate et al., 2016). In addition, future studies should test the differential effects found in this study to better understand their underlying mechanisms. Because the participating children in this study all received the same small-group intervention without additional specific individualization, it is precisely such individualization that could be implemented and analyzed in future studies. 


\section{DATA AVAILABILITY STATEMENT}

The raw data supporting the conclusion of this article will be made available by the authors, without undue reservation.

\section{ETHICS STATEMENT}

The studies involving human participants were reviewed and approved by the Ethics Committee of the University of Paderborn. Verbal informed consent to participate in this study was provided by the participants' legal guardian/next of kin.

\section{REFERENCES}

Abend, R., de Voogd, L., Salemink, E., Wiers, R. W., Pérez-Edgar, K., Fitzgerald, A., et al. (2018). Association between Attention Bias to Threat and Anxiety Symptoms in Children and Adolescents. Depress. Anxiety 35 (3), 229-238. doi:10.1002/da.22706

Ahrens-Eipper, S., Leplow, B., and Nelius, K. (2010). Mutig werden mit Til Tiger: Ein Trainingsprogramm für sozial unsichere Kinder [Becoming brave with Til the Tiger: a Training for Insecure Children] Göttingen: Hogrefe.

American Psychiatric Association (2013). Diagnostic and Statistical Manual of Mental Disorders. Washington DC: American Psychiatric Association, 5.

Bailey, R., and Jones, S. M. (2019). An Integrated Model of Regulation for Applied Settings. Clin. Child. Fam. Psychol. Rev. 22 (1), 2-23. doi:10.1007/ s10567-019-00288-y

Barlow, D. H., Nock, M., and Hersen, M. (2009). Single Case Experimental Designs: Strategies for Studying Behavior Change. Boston: Allyn \& Bacon.

Blanz, B., Remschmidt, H., Schmidt, M. H., and Warnke, A. (2006). Psychische Störungen im Kindes- und Jugendalter [Mental Disorders in Childhood and Adolescencce]. Stuttgart: Schattauer.

Broeren, S., Muris, P., Diamantopoulou, S., and Baker, J. R. (2013). The Course of Childhood Anxiety Symptoms: Developmental Trajectories and ChildRelated Factors in normal Children. J. Abnorm Child. Psychol. 41 (1), 81-95. doi:10.1007/s10802-012-9669-9

Brossart, D. F., Laird, V. C., and Armstrong, T. W. (2018). Interpreting Kendall's Tau and Tau-U for Single-Case Experimental Designs. Cogent Psychol. 5, 1518687. doi:10.1080/23311908.2018.1518687

Casale, G., Volpe, R. J., Briesch, A. M., Hennemann, T., and Grosche, M. (2021). Dependability of Direct Behavior Rating Single- and Multi-Item Scales across Raters and Occasions in Two School Subjects. Assess. Eff. Intervention 46, 143-154. doi:10.1177/1534508419836498

Casale, G., Volpe, R. J., Daniels, B., Hennemann, T., Briesch, A. M., and Grosche, M. (2018). Measurement Invariance of a Universal Behavioral Screener across Samples from the USA and Germany. Eur. J. Psychol. Assess. 34 (2), 87-100. doi:10.1027/1015-5759/a000447

Casale, G., Volpe, R. J., Hennemann, T., Briesch, A. M., Daniels, B., and Grosche, M. (2019). Konstruktvalidität eines universellen Screenings zur unterrichtsnahen und ökonomischen Diagnostik herausfordernden Verhaltens von Schüler_innen - eine Multitrait-Multimethod-Analyse. Z. für Pädagogische Psychol. 33 (1), 17-31. doi:10.1024/1010-0652/ a000232

Chafouleas, S. M., Sanetti, L. M. H., Kilgus, S. P., and Maggin, D. M. (2012). Evaluating Sensitivity to Behavioral Change Using Direct Behavior Rating Single-Item Scales. Exceptional Child. 78 (4), 491-505. doi:10.1177/ 001440291207800406

Christ, T. J., Riley-Tillman, T. C., and Chafouleas, S. M. (2009). Foundation for the Development and Use of Direct Behavior Rating (DBR) to Assess and Evaluate Student Behavior. Assess. Eff. Intervention 34 (4), 201-213. doi:10. $1177 / 1534508409340390$

\section{AUTHOR CONTRIBUTIONS}

All authors listed have made a substantial, direct, and intellectual contribution to the work and approved it for publication.

\section{ACKNOWLEDGMENTS}

We acknowledge support from the Open Access Publication Fund of the University of Wuppertal (Germany).

Clauss, J. A., and Blackford, J. U. (2012). Behavioral Inhibition and Risk for Developing Social Anxiety Disorder: A Meta-Analytic Study. J. Am. Acad. Child. Adolesc. Psychiatry 51, 1066-e1. doi:10.1016/j.jaac.2012. 08.002

Cohen, M., Buzinski, S. G., Armstrong-Carter, E., Clark, J., Buck, B., and Reuman, L. (2019). Think, Pair, Freeze: The Association between Social Anxiety and Student Discomfort in the Active Learning Environment. Scholarship Teach. Learn. Psychol. 5 (4), 265-277. doi:10.1037/stl0000147

Conely, L., Marchant, M., and Caldarelle, P. (2014). A Comparison of Teacher Perceptions and Research-Based Categories of Student Behavior Difficulties. Education 134 (4), 439-451.

Copeland, W. E., Angold, A., Shanahan, L., and Costello, E. J. (2014). Longitudinal Patterns of Anxiety from Childhood to Adulthood: The Great Smoky Mountains Study. J. Am. Acad. Child. Adolesc. Psychiatry 53 (1), 21-33. doi:10.1016/j.jaac.2013.09.017

Daniels, B., Volpe, R. J., Briesch, A. M., and Fabiano, G. A. (2014). Development of a Problem-Focused Behavioral Screener Linked to Evidence-Based Intervention. Sch. Psychol. Q. 29 (4), 438-451. doi:10.1037/spq0000100

De Los Reyes, A., Augenstein, T. M., Wang, M., Thomas, S. A., Drabick, D. A. G., Burgers, D. E., et al. (2015). The Validity of the Multi-Informant Approach to Assessing Child and Adolescent Mental Health. Psychol. Bull. 141 (4), 858-900. doi:10.1037/a0038498

Domitrovich, C. E., Bradshaw, C. P., Greenberg, M. T., Embry, D., Poduska, J. M., and Ialongo, N. S. (2010). Integrated Models of School-Based Prevention: Logic and Theory. Psychol. Sch. 47 (1), 71-88. doi:10.1002/ pits. 20452

Döpfner, M., Plück, J., and Kinnen, C. (2015). CBCL/6-18R, TRF/6-18R, YSR/11-18R: Deutsche Schulalter-Formen der Child Behavior Checklist von Thomas M. Achenbach. [German Language school-based version of Thomas M. Achenbach's Child Behavior Checklist]. Göttingen: Hogrefe.

Fox, N. A., Henderson, H. A., Marshall, P. J., Nichols, K. E., and Ghera, M. M. (2005). Behavioral Inhibition: Linking Biology and Behavior within a Developmental Framework. Annu. Rev. Psychol. 56, 235-262. doi:10.1146/annurev.psych.55. 090902.141532

Giannakakos, A. R., and Lanovaz, M. J. (2019). Using AB Designs with Nonoverlap Effect Size Measures to Support Clinical Decision-Making: A Monte Carlo Validation. Behav. Modif., 014544551986021. doi:10.1177/0145445519860219

Gresham, F. M. (2005). Methodological Issues in Evaluating Cognitive-Behavioral Treatments for Students with Behavioral Disorders. Behav. Disord. 30 (3), 213-225. doi:10.1177/019874290503000302

Henderson, H. A., Pine, D. S., and Fox, N. A. (2015). Behavioral Inhibition and Developmental Risk: A Dual-Processing Perspective. NeuropsychopharmacologyOfficial Publ. Am. Coll. Neuropsychopharmacol. 40 (1), 207-224. doi:10.1038/npp.2014.189

Hofmann, S. G., Asnaani, A., Vonk, I. J., Sawyer, A. T., and Fang, A. (2012). The Efficacy of Cognitive Behavioral Therapy: A Review of Meta-Analyses. Cognit. Ther. Res. 36 (5), 427-440. doi:10.1007/s10608-012-9476-1

Huber, L., Plötner, M., In-Albon, T., Stadelmann, S., and Schmitz, J. (2019). The Perspective Matters: A Multi-Informant Study on the Relationship between Social-Emotional Competence and Preschoolers' Externalizing and 
Internalizing Symptoms. Child. Psychiatry Hum. Dev. 50 (6), 1021-1036. doi:10.1007/s10578-019-00902-8

Huitema, B. E., and Mckean, J. W. (2000). Design Specification Issues in Time-Series Intervention Models. Educ. Psychol. Meas. 60 (1), 38-58. doi:10.1177/ 00131640021970358

Hyman, I. A., Wojtowicz, A., Lee, K. D., Haffner, M. E., Fiorello, C. A., Storlazzi, J. J., et al. (1998). School-based Methylphenidate Placebo Protocols: Methodological and Practical Issues. J. Learn. Disabil. 31 (6), 581-614. doi:10.1177/002221949803100609

Jacobson, N. S., and Truax, P. (1992). "Clinical Significance : A Statistical Approach to Defining Meaningful Change in Psychotherapy Research," in Methodological Issues \& Strategies in Clinical Research. Editor A. E. Kazdin (Washington, DC: American Psychological Association), 631-648. doi:10.1037/10109-042

Kessler, R. C., Avenevoli, S., McLaughlin, K. A., Green, J. G., Lakoma, M. D., Petukhova, M., et al. (2012). Lifetime Co-morbidity of DSM-IV Disorders in the US National Comorbidity Survey Replication Adolescent Supplement (NCS-A). Psychol. Med. 42 (9), 1997-2010. doi:10.1017/ S0033291712000025

Kilgus, S. P., van Wie, M. P., Sinclair, J. S., Riley-Tillman, T. C., and Herman, K. C. (2019). Developing a Direct Rating Behavior Scale for Depression in Middle School Students. Sch. Psychol. Q. 34 (1), 86-95. doi:10.1037/ spq0000263

Klasen, F., Petermann, F., Meyrose, A.-K., Barkmann, C., Otto, C., Haller, A.-C., et al. (2016). Verlauf psychischer Auffälligkeiten von Kindern und Jugendlichen. Kindheit Und Entwicklung 25 (1), 10-20. doi:10.1026/09425403/a000184

Kleberg, J. L., Högström, J., Sundström, K., Frick, A., and Serlachius, E. (2021). Delayed Gaze Shifts Away from Others' Eyes in Children and Adolescents with Social Anxiety Disorder. J. Affect Disord. 278, 280-287. doi:10.1016/j.jad.2020. 09.022

Krull, J., Wilbert, J., and Hennemann, T. (2018). Does Social Exclusion by Classmates lead to Behaviour Problems and Learning Difficulties or Vice Versa? A Cross-Lagged Panel Analysis. Eur. J. Spec. Needs Edu. 33 (2), 235-253. doi:10.1080/08856257.2018.1424780

Luis-Joaquin, G. L., Lourdes, E. F., and José A, M. M. (2020). Behavioral Inhibition in Childhood as A Risk Factor for Development of Social Anxiety Disorder: A Longitudinal Study. Int. J. Environ. Res. Public Health 17, 11. doi:10.3390/ ijerph17113941

Maggin, D. M., Cook, B. G., and Cook, L. (2018). Using Single-Case Research Designs to Examine the Effects of Interventions in Special Education. Learn. Disabilities Res. Pract. 33 (4), 182-191. doi:10.1111/ldrp.12184

Manolov, R., and Moeyaert, M. (2017). Recommendations for Choosing SingleCase Data Analytical Techniques. Behav. Ther. 48 (1), 97-114. doi:10.1016/j. beth.2016.04.008

Matta, M., Volpe, R. J., Briesch, A. M., and Owens, J. S. (2020). Five Direct Behavior Rating Multi-Item Scales: Sensitivity to the Effects of Classroom Interventions. J. Sch. Psychol. 81, 28-46. doi:10.1016/j.jsp.2020.05.002

Mayer, M., Lochman, J., and van Acker, R. (2005). Introduction to the Special Issue: Cognitive-Behavioral Interventions with Students with EBD. Behav. Disord. 303, 197-212. doi:10.1177/019874290503000306

Merikangas, K. R., He, J. P., Burstein, M., Swanson, S. A., Avenevoli, S., Cui, L., et al. (2010). Lifetime Prevalence of Mental Disorders in U.S. Adolescents: Results from the National Comorbidity Survey Replication--Adolescent Supplement (NCS-A). J. Am. Acad. Child. Adolesc. Psychiatry 49 (10), 980-989. doi:10.1016/ j.jaac.2010.05.017

Parker, R. I., and Vannest, K. (2009). An Improved Effect Size for Single-Case Research: Nonoverlap of All Pairs. Behav. Ther. 40 (4), 357-367. doi:10.1016/j. beth.2008.10.006

Parker, R. I., Vannest, K. J., Davis, J. L., and Sauber, S. B. (2011). Combining Nonoverlap and Trend for Single-Case Research: Tau-U. Behav. Ther. 42 (2), 284-299. doi:10.1016/j.beth.2010.08.006

Pergamin-Hight, L., Pine, D. S., Fox, N. A., and Bar-Haim, Y. (2016). Attention Bias Modification for Youth with Social Anxiety Disorder. J. Child. Psychol. Psychiatry 57 (11), 1317-1325. doi:10.1111/jcpp.12599

R Core Team (2018). R [Computer Software]. Vienna. Available at: https://www.Rproject.org (Accessed July 22, 2021).
Rapee, R. M., and Spence, S. H. (2004). The Etiology of Social Phobia: Empirical Evidence and an Initial Model. Clin. Psychol. Rev. 24 (7), 737-767. doi:10.1016/ j.cpr.2004.06.004

Riley-Tillman, T. C., Burns, M. K., and Kilgus, S. P. (2020). Evaluating Educational Interventions: Single-Case Design for Measuring Response to Intervention. New York: Guilford.

Rindskopf, D. M., and Ferron, J. M. (2014). "Using Multilevel Models to Analyze Single-Case Design Data," in Single-case Intervention Research: Methodological and Statistical Advances. Editors D. M. Rindskopf, J. M. Ferron, T. R. Kratochwill, and J. R. Levin (Washington DC: American Psychological Association), 221-246.

Sandstrom, A., Uher, R., and Pavlova, B. (2020). Prospective Association between Childhood Behavioral Inhibition and Anxiety: A Meta-Analysis. J. Abnorm. Child. Psychol. 48 (1), 57-66. doi:10.1007/s10802-019-00588-5

Scaini, S., Belotti, R., Ogliari, A., and Battaglia, M. (2016). A Comprehensive MetaAnalysis of Cognitive-Behavioral Interventions for Social Anxiety Disorder in Children and Adolescents. J. Anxiety Disord. 42, 105-112. doi:10.1016/j.janxdis. 2016.05.008

Schoenfeld, N. A., and Mathur, S. R. (2009). Effects of Cognitive-Behavioral Intervention on the School Performance of Students with Emotional or Behavioral Disorders and Anxiety. Behav. Disord. 34 (4), 184-195. doi:10. 1177/019874290903400401

Segool, N. K., and Carlson, J. S. (2008). Efficacy of Cognitive-Behavioral and Pharmacological Treatments for Children with Social Anxiety. Depress. Anxiety 25 (7), 620-631. doi:10.1002/da.20410

Smith, R. L., Eklund, K., and Kilgus, S. P. (2018). Concurrent Validity and Sensitivity to Change of Direct Behavior Rating Single-Item Scales (DBRSIS) within an Elementary Sample. Sch. Psychol. Q. 33 (1), 83-93. doi:10.1037/ spq0000209

Tate, R. L., Perdices, M., Rosenkoetter, U., McDonald, S., Togher, L., Shadish, W., et al. (2016). The Single-Case Reporting Guideline in BEhavioural Interventions (SCRIBE) 2016: Explanation and Elaboration. Arch. Scientific Psychol. 4 (1), 10-31. doi:10.1037/arc0000027

van den Noortgate, W., and Onghena, P. (2003). Hierarchical Linear Models for the Quantitative Integration of Effect Sizes in Single-Case Research. Behav. Res. Methods Instrum Comput. 35 (1), 1-10. doi:10.3758/ BF03195492

Vannest, K. J., and Ninci, J. (2015). Evaluating Intervention Effects in SingleCase Research Designs. J. Couns. Dev. 93 (4), 403-411. doi:10.1002/jcad. 12038

Volpe, R., and Fabiano, G. (2013). Daily Behavior Report Cards: An EvidenceBased System of Assessment and Intervention. New York, NY: Guilford Press.

Volpe, R. J., and Briesch, A. M. (2015). Multi-item Direct Behavior Ratings: Dependability of Two Levels of Assessment Specificity. Sch. Psychol. Q. 30 (3), 431-442. doi:10.1037/spq0000115

Volpe, R. J., Casale, G., Mohiyeddini, C., Grosche, M., Hennemann, T., Briesch, A. M., et al. (2018). A Universal Behavioral Screener Linked to Personalized Classroom Interventions: Psychometric Characteristics in a Large Sample of German Schoolchildren. J. Sch. Psychol. 66, 25-40. doi:10.1016/j.jsp.2017. 11.003

Volpe, R. J., Yeung, T. S., Casale, G., Krull, J., Briesch, A. M., and Henneman, T. (2020). Evaluation of a German Language School-Based Universal Screening for Student Social, Emotional, and Behavioral Risk. Int. J. Sch. Educ. Psychol. 9, S10-S20. doi:10.1080/21683603.2020.1717699

von der Embse, N. P., Scott, E.-C., and Kilgus, S. P. (2015). Sensitivity to Change and Concurrent Validity of Direct Behavior Ratings for Academic Anxiety. Sch. Psychol. Q. 30 (2), 244-259. doi:10.1037/spq0000083

Wilbert, J., Bosch, J., and Lüke, T. (2021). Validity and Judgment Bias in Visual Analysis of Single-Case Data. ijrld, 13-24. doi:10.28987/5.1.13

Wilbert, J., and Lüke, T. (2019). Scan: Single-Case Data Analyses for Single and Multiple Baseline Designs. R package version 0.40. Available at: https://CRAN. R-project.org/package=scan (Accessed July 22, 2021).

Woodward, L. J., and Fergusson, D. M. (2001). Life Course Outcomes of Young People with Anxiety Disorders in Adolescence. J. Am. Acad. Child. Adolesc. Psychiatry 40 (9), 1086-1093. doi:10.1097/00004583-20010900000018 
Zhu, X., Huebner, E. S., and Tian, L. (2019). A Person-Centered Longitudinal Analysis of Adolescents' Loneliness and Social Anxiety: Clusters, Predictors, and Outcomes. Sch. Psychol. 34 (5), 576-589. doi:10.1037/spq0000328

Conflict of Interest: The authors declare that the research was conducted in the absence of any commercial or financial relationships that could be construed as a potential conflict of interest.

Publisher's Note: All claims expressed in this article are solely those of the authors and do not necessarily represent those of their affiliated organizations, or those of the publisher, the editors and the reviewers. Any product that may be evaluated in this article, or claim that may be made by its manufacturer, is not guaranteed or endorsed by the publisher.

Copyright $\odot 2022$ Casale and Haarhoff. This is an open-access article distributed under the terms of the Creative Commons Attribution License (CC BY). The use, distribution or reproduction in other forums is permitted, provided the original author(s) and the copyright owner(s) are credited and that the original publication in this journal is cited, in accordance with accepted academic practice. No use, distribution or reproduction is permitted which does not comply with these terms. 